University of Nebraska - Lincoln

DigitalCommons@University of Nebraska - Lincoln

Faculty Publications, Department of Physics and Astronomy

Research Papers in Physics and Astronomy

2020

\title{
Interfacial contributions to spin-orbit torque and magnetoresistance in ferromagnet/heavy-metal bilayers
}

Kirill Belashchenko

University of Nebraska - Lincoln, belashchenko@unl.edu

Alexey Kovalev

University of Nebraska - Lincoln, alexey.kovalev@unl.edu

Mark van Schilfgaarde

King's College London, mark.van_schilfgaarde@kcl.ac.uk

Follow this and additional works at: https://digitalcommons.unl.edu/physicsfacpub

Part of the Condensed Matter Physics Commons

Belashchenko, Kirill; Kovalev, Alexey; and van Schilfgaarde, Mark, "Interfacial contributions to spin-orbit torque and magnetoresistance in ferromagnet/heavy-metal bilayers" (2020). Faculty Publications, Department of Physics and Astronomy. 306.

https://digitalcommons.unl.edu/physicsfacpub/306

This Article is brought to you for free and open access by the Research Papers in Physics and Astronomy at DigitalCommons@University of Nebraska - Lincoln. It has been accepted for inclusion in Faculty Publications, Department of Physics and Astronomy by an authorized administrator of DigitalCommons@University of Nebraska - Lincoln. 


\title{
Interfacial contributions to spin-orbit torque and magnetoresistance in ferromagnet/heavy-metal bilayers
}

\author{
K. D. Belashchenko $\odot,{ }^{1}$ Alexey A. Kovalev, ${ }^{1}$ and M. van Schilfgaarde ${ }^{2}$ \\ ${ }^{1}$ Department of Physics and Astronomy and Nebraska Center for Materials and Nanoscience, University of Nebraska-Lincoln, \\ Lincoln, Nebraska 68588, USA \\ ${ }^{2}$ Department of Physics, King's College London, Strand, London WC2R 2LS, United Kingdom
}

(Received 23 August 2019; published 17 January 2020)

\begin{abstract}
The thickness dependence of spin-orbit torque and magnetoresistance in ferromagnet/heavy-metal bilayers is studied using the first-principles nonequilibrium Green's function formalism combined with the Anderson disorder model. A systematic expansion in orthogonal vector spherical harmonics is used for the angular dependence of the torque. The dampinglike torque in $\mathrm{Co} / \mathrm{Pt}$ and $\mathrm{Co} / \mathrm{Au}$ bilayers can be described as a sum of the spin-Hall contribution, which increases with thickness in agreement with the spin-diffusion model, and a comparable interfacial contribution. The magnetoconductance in the plane perpendicular to the current in $\mathrm{Co} / \mathrm{Pt}$ bilayers is of the order of a conductance quantum per interfacial atom, exceeding the prediction of the spin-Hall model by more than an order of magnitude. This suggests that the "spin-Hall magnetoresistance," similarly to the dampinglike torque, has a large interfacial contribution unrelated to the spin-Hall effect.
\end{abstract}

DOI: 10.1103/PhysRevB.101.020407

Introduction. Magnetization in nanoelectronic devices can be manipulated by angular momentum transfer [1,2]. A spinorbit torque (SOT) [3-5] is a manifestation of such transfer driven by spin-orbit coupling in ferromagnet/heavy-metal (FM/HM) bilayers, which is also responsible for anisotropic transport properties such as spin-Hall magnetoresistance (SMR) [6,7]. SOT and SMR involve complex processes both in the bulk and at the interface [5,7-11]. The role played by spin currents in angular momentum transfer is still being actively investigated both experimentally [12,13] and theoretically $[14,15]$. However, it is also clear that SOT is not fully determined by spin currents because magnetization can exchange angular momentum with the lattice.

SMR is usually explained by interfacial absorption of the spin-Hall current incident from the bulk of the heavy metal [16]. It is also common to attribute dampinglike SOT to the spin-Hall effect [17] originating in the bulk of the heavy metal $[4,18,19]$ and fieldlike SOT to the inverse spin-galvanic effect [20-22] at the interface [3,23-26]. However, dampinglike SOT can also be generated at the interface [8,9,27-29] without any spin-polarized current incident from the bulk of the HM layer. Besides, the layers in FM/HM bilayers are usually about a nanometer thick or even less. Thus, the distinction between interfacial and bulk contributions to SOT is not well defined, which prompts a fully quantum-mechanical treatment of the whole device [30]. This point of view is supported by experiments suggesting a competition between interfacial and bulk contributions to dampinglike SOT [31-34] and by $a b$ initio calculations hinting at the importance of interfacial contributions [30,35].

Most $a b$ initio calculations of SOT have been performed using the linear response method with phenomenological broadening for the Green's functions [36] and did not reveal the interfacial contribution to dampinglike SOT [37]. However, given that various bulk, interfacial, and disorder effects can influence SOT and SMR in interconnected ways, it is advantageous to treat them all on equal footing. A suitable computational technique based on the nonequilibrium Green's function (NEGF) approach [38,39] within the tight-binding linear muffin-tin orbital (LMTO) method [40,41], with an explicit treatment of disorder, has recently become available [30]. In this Rapid Communication, we employ this technique to study the thickness dependence of SOT and SMR in FM/HM bilayers. For the leading dampinglike SOT, we find a contribution increasing with thickness in agreement with the spin-diffusion model and a comparable interfacial contribution that survives in the limit of zero thickness. We further identify the interfacial contribution to magnetoresistance which exceeds the expected spin-Hall contribution by more than an order of magnitude.

Thickness dependence of SOT. The technical details of the $a b$ initio NEGF calculations are similar to Ref. [30], except that here we represent the angular dependence of SOT using the complete orthonormal basis of vector spherical harmonics, as described in the Appendix. The expansion is generally given by Eq. (A6), and the three harmonics relevant here are $\mathbf{Z}_{1,-1}^{(1)}, \mathbf{Z}_{1,-1}^{(2)}$, and $\mathbf{Z}_{2,1}^{(1)}$; their relation to the familiar dampinglike, fieldlike, and planar-Hall-like SOT terms is given in Eqs. (A7)-(A9). Only the Fermi-surface contribution is considered here, because the Fermi-sea term is considerably smaller at room temperature [30]. We employ the Anderson disorder model with a uniformly distributed random potential $V_{i},-V_{m}<V_{i}<V_{m}$, applied on each lattice site $i$ with an amplitude $V_{m}=0.77$ or $1.09 \mathrm{eV}$, which results in resistivities typically observed in FM/HM bilayers (see Table I). The SOT is calculated from the nonequilibrium spin density matrix on each atom. 
TABLE I. Parameters of the fits [Eq. (1)] for the thickness dependence of the damping-like SOT coefficient $C_{1,-1}^{(1)}$. The effective resistivities $\bar{\rho}$ are given for the $\mathrm{Co}(4 \mathrm{ML}) / \mathrm{HM}(12 \mathrm{ML})$ bilayers.

\begin{tabular}{lccrrrr}
\hline \hline $\mathrm{FM} / \mathrm{HM}$ & $V_{m}(\mathrm{eV})$ & $\bar{\rho}(\mu \Omega \mathrm{cm})$ & $\tau_{0}(\mathrm{~ns} / \mathrm{m})$ & $\tau_{\mathrm{SH}}(\mathrm{ns} / \mathrm{m})$ & $l_{s f}(\mathrm{~nm})$ & $\theta_{\mathrm{SH}}$ \\
\hline $\mathrm{Co} / \mathrm{Pt}$ & 1.09 & 27.2 & 110.3 & 96.5 & 1.94 & 0.027 \\
& 0.77 & 17.7 & 133.2 & 97.5 & 2.43 & 0.018 \\
$\mathrm{Co} / \mathrm{Au}$ & 1.09 & 6.4 & 54.6 & 108.9 & 3.61 & 0.007 \\
& 0.77 & 4.1 & 58.2 & 81.1 & 3.38 & 0.003 \\
\hline \hline
\end{tabular}

Figure 1 shows the dependence of SOT in Co/Pt and $\mathrm{Co} / \mathrm{Au}$ bilayers on the thickness of the heavy-metal layer $d_{N}$ measured in monolayers (ML). The dampinglike SOT coefficient $C_{1,-1}^{(1)}$ is well described by the function

$$
C_{1,-1}^{(1)}=\tau_{0}+\tau_{\mathrm{SH}}\left[1-\operatorname{sech}\left(d_{N} / l_{s f}\right)\right],
$$

with the parameters listed in Table I, where $l_{s f}$ is the spindiffusion length in the heavy-metal layer, $\tau_{0}$ represents the
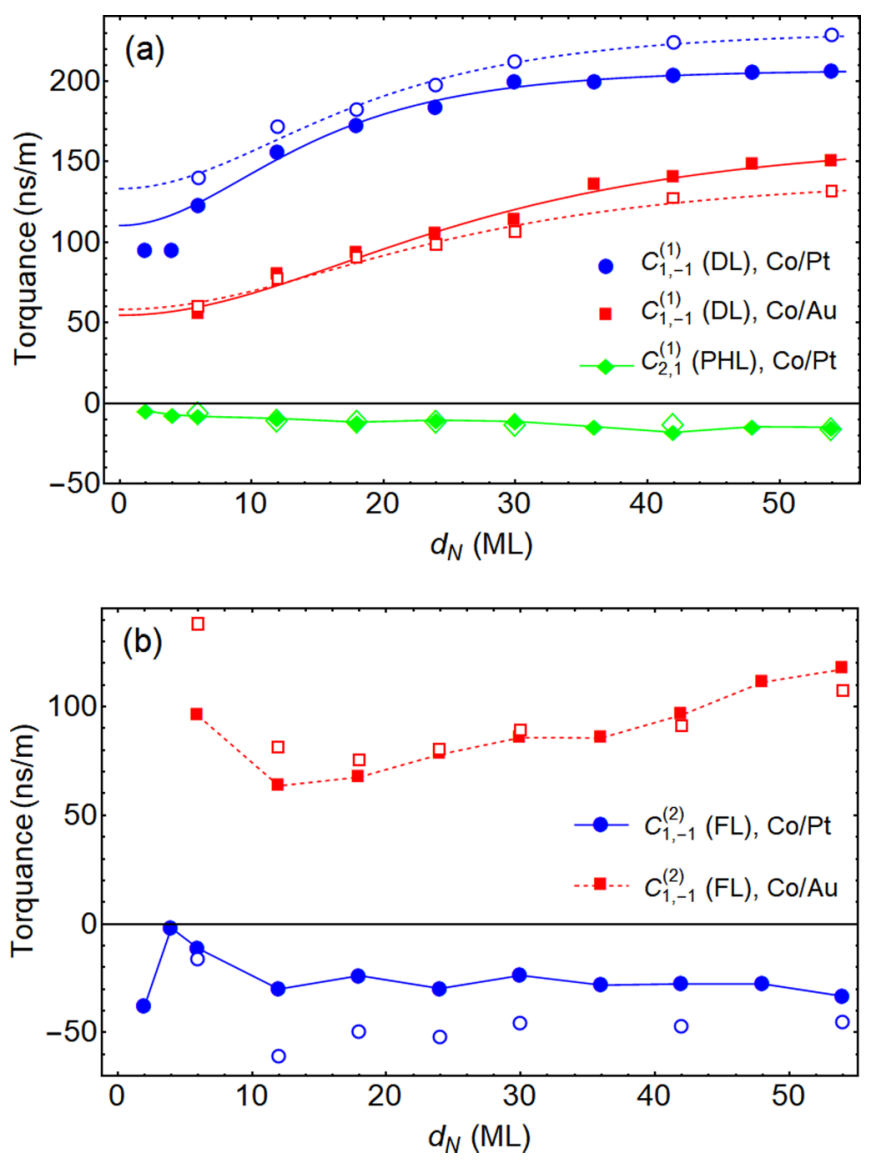

FIG. 1. Dependence of the SOT coefficients on the thickness $d_{N}$ of the heavy-metal layer in $\mathrm{Co} / \mathrm{Pt}$ and $\mathrm{Co} / \mathrm{Au}$ bilayers. (a) Dampinglike (DL) $C_{1,-1}^{(1)}$ and planar-Hall-like (PHL) $C_{2,1}^{(1)}$ coefficients. (b) Fieldlike (FL) coefficient $C_{1,-1}^{(2)}$. Blue (red) circles (squares): $C_{1,-1}^{(1)}$ or $C_{1,-1}^{(2)}$ for $\mathrm{Co} / \mathrm{Pt}(\mathrm{Co} / \mathrm{Au})$; green lines and diamonds: $C_{2,1}^{(1)}$ for $\mathrm{Co} / \mathrm{Pt}$. Solid (open) symbols: $V_{m}=1.09 \mathrm{eV}(0.77 \mathrm{eV})$. Blue (red) lines in (a): Fits of the data for $\mathrm{Co} / \mathrm{Pt}(\mathrm{Co} / \mathrm{Au})$ at $V_{m}=1.09 \mathrm{eV}$ (see text). thickness-independent interfacial contribution to SOT, and $\tau_{\mathrm{SH}}$ is the conventional spin-Hall-generated part that follows from spin-diffusion theory [4]. However, the fitting (1) should be approached with care, because it assumes a geometrical interface between homogeneous bulk regions and ignores thickness-dependent perturbations and finite-size effects in the electronic structure of the bilayer.

Importantly, we find that $\tau_{0}$, which can appear due to interface scattering $[8,9]$, is comparable with $\tau_{\mathrm{SH}}$ in both systems and is especially large in Co/Pt. The value of $\tau_{\mathrm{SH}}$ is similar in $\mathrm{Co} / \mathrm{Pt}$ and $\mathrm{Co} / \mathrm{Au}$, while the spin-diffusion length is larger in $\mathrm{Co} / \mathrm{Au}$.

The influence of disorder strength on the dampinglike SOT can be seen by comparing the results for $V_{m}=1.09$ and $0.77 \mathrm{eV}$ in Fig. 1(a) (solid and open symbols, respectively) and Table I. The behavior is different in $\mathrm{Co} / \mathrm{Pt}$ and $\mathrm{Co} / \mathrm{Au}$ bilayers. In Co/Pt, increasing $V_{m}$ from 0.77 to $1.09 \mathrm{eV}$ reduces $l_{s f}$ and $\tau_{0}$, both by about $20 \%$, while $\tau_{\mathrm{SH}}$ remains unchanged. This suggests that the spin-Hall contribution to dampinglike $\mathrm{SOT}$ in $\mathrm{Co} / \mathrm{Pt}$ is dominated by a combination of intrinsic and side-jump mechanisms, which are insensitive to the relaxation time [17]. In contrast, stronger disorder in $\mathrm{Co} / \mathrm{Au}$ leads to a $34 \%$ increase in $\tau_{\mathrm{SH}}$ while the changes in $l_{s f}$ and $\tau_{0}$ are within the margin of error. The dependence of $\tau_{\mathrm{SH}}$ on $V_{m}$ in $\mathrm{Co} / \mathrm{Au}$ suggests that the skew-scattering contribution, which is proportional to the relaxation time [17], is not negligible and negative in this system. The suppression of the skew scattering contribution in $\mathrm{Co} / \mathrm{Pt}$ is likely due to the much larger resistivity in this system compared to $\mathrm{Co} / \mathrm{Au}$.

Interestingly, $l_{s f}$ does not increase with increasing conductivity in $\mathrm{Co} / \mathrm{Au}$, suggesting that the Dyakonov-Perel mechanism of spin relaxation, which was shown to be dominant in some supported metallic films [42], can survive even in the presence of a proximate ferromagnetic layer. This mechanism is expected to be more important in $\mathrm{Co} / \mathrm{Au}$ compared to $\mathrm{Co} / \mathrm{Pt}$, because the magnetic proximity effect in $\mathrm{Au}$ is much weaker than in Pt.

We have previously found [30] that spin-orbit coupling on the Co atoms has no effect on the total dampinglike SOT in the Co(6 ML)/Pt(6 ML) bilayer. The same statement holds for $\mathrm{Co}(4 \mathrm{ML}) / \mathrm{Pt}(30 \mathrm{ML})$ and $\mathrm{Co}(4 \mathrm{ML}) / \mathrm{Au}(30 \mathrm{ML})$ bilayers, suggesting that the spin-Hall effect in Co [14] does not contribute materially to dampinglike SOT.

Table I also lists the effective spin-Hall angle in the heavy metal estimated as

$$
\theta_{\mathrm{SH}}=\sqrt{\frac{3}{8 \pi}} \frac{2 e}{\hbar} \tau_{\mathrm{SH}} \bar{\rho} \frac{M}{A},
$$

where $M / A$ is the total magnetic moment per area of the film, and the numerical factor comes from Eq. (A7). Small $\theta_{\mathrm{SH}}$ in $\mathrm{Co} / \mathrm{Au}$ is due to the low resistivity of Au compared to Pt. The values for $\mathrm{Co} / \mathrm{Pt}$ are smaller compared to the experimental measurements; for example, $\theta_{\mathrm{SH}} \approx 0.06$ was reported in Ref. [4]. However, including the interfacial contribution $\tau_{0}$ would result in $\theta_{\mathrm{SH}} \approx 0.06$, which may increase further in more resistive films. We also note that the spin-Hall conductivity estimated from $\tau_{\mathrm{SH}}$ assuming the spin-Hall current is fully absorbed by the magnetization is similar to the result of the Berry-phase calculation at zero temperature [43]. 
The planar-Hall-like term $C_{2,1}^{(1)}$ in $\mathrm{Co} / \mathrm{Pt}$ is roughly proportional to the leading dampinglike coefficient $C_{1,-1}^{(1)}$ and amounts to about $-7 \%$ of it. This term can be isolated experimentally by measuring current-induced magnetization damping in the $x z$ plane $[30,44]$. It is seen from Fig. 1(a) that disorder strength has little influence on $C_{2,1}^{(1)}$, consistent with its attribution to vertex corrections [30]. In $\mathrm{Co} / \mathrm{Au}$ the planar-Hall-like term is positive, does not exceed $5 \mathrm{~ns} / \mathrm{m}$, and is not shown in Fig. 1(a).

The thickness dependence of the fieldlike SOT $C_{1,-1}^{(2)}$ is shown in Fig. 1(b). This term exhibits sharp variations at small thicknesses, which likely reflect the sensitivity of the underlying mechanism to the electronic structure near the interface. At larger thicknesses the fieldlike SOT converges to a moderate value in $\mathrm{Co} / \mathrm{Pt}$, but in $\mathrm{Co} / \mathrm{Au}$ it is comparable to the dampinglike SOT and keeps growing at large thicknesses. This growth may indicate that the fieldlike SOT in $\mathrm{Co} / \mathrm{Au}$ has a contribution that is associated with the spin-Hall effect in $\mathrm{Au}$.

The effect of disorder strength on the fieldlike SOT is also different in $\mathrm{Co} / \mathrm{Pt}$ and $\mathrm{Co} / \mathrm{Au}$ bilayers. While stronger disorder tends to suppress $C_{1,-1}^{(2)}$ in $\mathrm{Co} / \mathrm{Pt}$, which is consistent with the inverse spin galvanic effect (ISGE), it has hardly any effect on it in $\mathrm{Co} / \mathrm{Au}$. This feature is due to the cancellation of disorder-induced trends in different bands. Indeed, if spinorbit coupling is turned off on the Co atoms, the fieldlike $C_{1,-1}^{(2)}$ coefficient at $d_{N}=30 \mathrm{ML}$ increases from 86 to $150 \mathrm{~ns} / \mathrm{m}$ at $V_{m}=1.09 \mathrm{eV}$, and from 89 to $226 \mathrm{~ns} / \mathrm{m}$ at $0.77 \mathrm{eV}$. Thus, the contributions to the fieldlike torque from spin-orbit coupling on Co and Pt atoms have opposite signs and both decrease with increasing disorder, as expected for ISGE.

Magnetoresistance. The term spin-Hall magnetoresistance (SMR) refers to the reduction of the resistance of a FM/HM bilayer whose magnetization is aligned parallel to the interface and perpendicular to the current flow, i.e., along the $\hat{y}$ axis. Measurements of the magnetoresistance in fully metallic bilayers [45] including $\mathrm{Co} / \mathrm{Pt}$ [46] have been attributed to the spin-Hall mechanism.

The phenomenological theory of SMR [16], which was introduced for a bilayer with an insulating FM layer, describes the effect of spin-dependent interfacial scattering of the spinHall current incident from the heavy-metal layer and predicts the $\cos ^{2} \theta_{y}$ angular dependence of the magnetoresistance. However, such magnetoresistance can also arise due to purely interfacial mechanisms [47-49]. Metallic FM/HM bilayers also exhibit anisotropic magnetoresistance (AMR) with the $\cos ^{2} \theta_{x}$ angular dependence contributed by the metallic FM layer. In addition, the dependence of the interfacial electronic structure on the orientation of the magnetization [50] should result in an interfacial anomalous magnetoresistance (IAMR) with the $\cos ^{2} \theta_{z}$ angular dependence. IAMR is similar to the tunneling anisotropic magnetoresistance [51] but occurs in the current-in-plane geometry.

Because the three functions $\cos ^{2} \theta_{\alpha}$ representing the angular dependence of SMR, AMR, and IAMR are linearly dependent, there are, in fact, only two independent parameters that can be extracted from experiment, and different mechanisms cannot be uniquely separated from the angular dependence. On the other hand, only the SMR mechanism is due to the
spin-Hall effect in the bulk of the heavy-metal layer, and it comes with a characteristic dependence on its thickness $d_{N}[16]$.

We introduce the reduced conductance $g(\hat{m})=L G(\hat{m}) / w$ in the Ohmic limit (large $L$ and $w$ ), where $G(\hat{m})$ is the conductance, $L$ the length, and $w$ the width of the bilayer, and write it as

$$
g\left(\hat{m}, d_{N}\right)=g_{F}\left(\hat{m}, d_{N}\right)+\sigma_{N} d_{N},
$$

where $\sigma_{N}$ is the conductivity and $d_{N}$ the thickness of the normal metal. The two terms on the right-hand side represent, respectively, the angular-dependent contribution of the FM layer (including the interface), and the angular-independent bulk contribution of the normal metal. The dependence of $g_{F}$ on $d_{N}$ can come both from the spin-Hall contribution and from Friedel oscillations and quantum-well-like effects in other mechanisms. Of course, the angular dependence of $g_{F}$ also includes the contribution of AMR.

Defining the difference $\Delta_{\mu \nu} g\left(d_{N}\right)=g\left(\hat{\mu}, d_{N}\right)-g\left(\hat{v}, d_{N}\right)$, where $\hat{\mu}, \hat{v}$ are some chosen orientations of $\hat{m}$, we have $\Delta_{\mu \nu} g\left(d_{N}\right)=\Delta_{\mu \nu} g_{F}\left(d_{N}\right)$. The quantity $\Delta_{y z} g / g$ is usually reported as SMR. Note that $\Delta_{\mu \nu} g$ is expected to saturate at large thicknesses. The prediction of the spin-Hall theory $[16,45]$ for the angular and thickness dependence of $\Delta_{y z} g$ can be compared with the results of ab initio calculations.

We compute $g(\hat{x}), g(\hat{y})$, and $g(\hat{z})$ for $\mathrm{Co} / \mathrm{Pt}$ bilayers with a varying thickness $d_{N}$ of the Pt layer while keeping the Co layer $4 \mathrm{ML}$ thick. For each value of $d_{N}$, we calculate the conductance $G$ for a fixed width $w$ and $L=60,90, \ldots, 240 \mathrm{ML}$, taking several hundred disorder configurations for each $L$. The data set for the given $d_{N}$ and $w$ is then fitted to the function $G(L)=\left(R_{0}+L g / w\right)^{-1}$, which provides the value of $g$ for the given $\hat{m}$. Finally, we find $\Delta_{y z} g\left(d_{N}\right)$ and $\Delta_{y x} g\left(d_{N}\right)$. Most of the calculations were performed with $w=12 \mathrm{ML}$, but for $d_{N}=4$ and $12 \mathrm{ML}$ we also considered $w=16 \mathrm{ML}$ to check convergence with respect to $w$.

The results of the calculations of $\Delta_{y z} g\left(d_{N}\right)$ and $\Delta_{y x} g\left(d_{N}\right)$, along with their standard deviations, are shown in Fig. 2. The magnitude of $\Delta_{y x} g$, which is influenced by SMR and AMR but not IAMR, appears to be thickness independent apart from relatively small oscillations. $\Delta_{y z} g$, which is influenced by SMR and IAMR but not AMR, is of the order of one conductance quantum, which is well above the margin of error, but its thickness dependence is obscured by the relatively large error bars.

The spin-Hall theory $[16,45]$ predicts the following spinHall contribution to $\Delta_{y z} g$, where we assume the magnetic layer is sufficiently thin so that the shunting effect can be neglected,

$$
\Delta_{y z} g_{\mathrm{SH}}=\frac{\theta_{\mathrm{SH}}^{2}}{\bar{\rho}} \tanh ^{2}\left(d_{N} / 2 l_{s f}\right) \tanh \left(d_{N} / l_{s f}\right) .
$$

The values of $\theta_{\mathrm{SH}}, l_{s f}$, and $\bar{\rho}$ obtained from SOT calculations can be found in Table I. The dashed line in Fig. 2 shows the resulting $\Delta_{y z} g_{\mathrm{SH}}\left(d_{N}\right)$ scaled by a factor of 10. It is clear that the spin-Hall mechanism $[16,45]$ is too weak, by more than an order of magnitude, to account for the calculated 


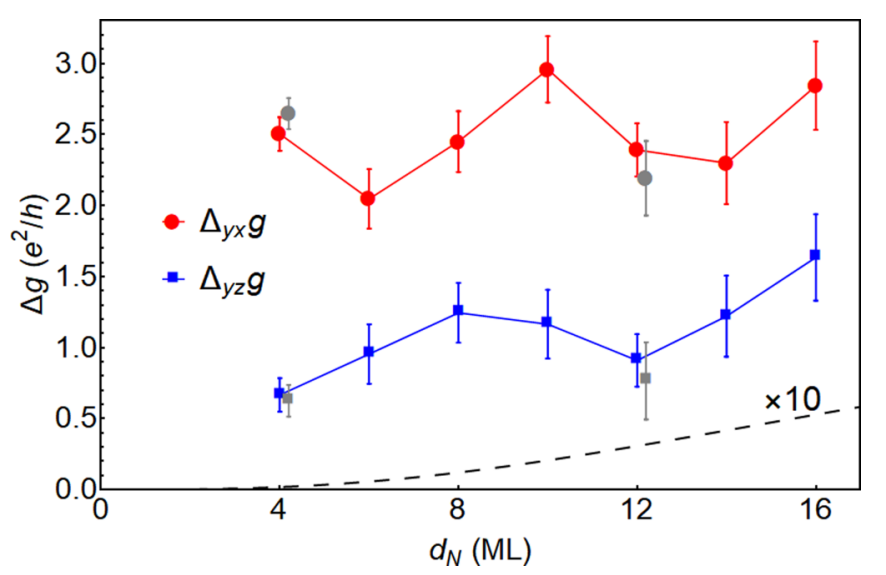

FIG. 2. Dependence of the magnetoconductances $\Delta_{y x} g$ (red symbols) and $\Delta_{y z} g$ (blue symbols) in Co/Pt bilayers on the thickness of the Pt layer calculated at $w=12 \mathrm{ML}$. Gray symbols: Convergence tests at $w=16 \mathrm{ML}$. The disorder strength is $V_{m}=1.09 \mathrm{eV}$. The error bars show the standard deviation. Dashed line: The prediction of Eq. (4) with the parameters for $\mathrm{Co} / \mathrm{Pt}$ at $V_{m}=1.09 \mathrm{eV}$ from Table I, scaled by a factor of 10 .

magnetoconductance $\Delta_{y z} g \sim e^{2} / h$. The effect can, therefore, be entirely due to interfacial mechanisms.

The growth of $\Delta g_{y z} / g$ at small thicknesses $[45,46]$ is the main evidence in favor of the spin-Hall theory of magnetoresistance in FM/HM bilayers. However, this inference assumes that the interface is well formed and has the same properties for all $d_{N}$. In a practical device, this assumption can fail once $d_{N}$ is reduced to a few monolayers, which is comparable to both the typical interfacial roughness and to the screening length of the metal. Thus, the region of linear growth of $\Delta g_{y z} / g$ could indicate the typical thickness at which the continuous and homogeneous heavy-metal film is formed during deposition. Interfacial roughness sets the natural scale for this thickness.

Conclusions. The results of $a b$ initio calculations suggest that the dampinglike SOT in $\mathrm{Co} / \mathrm{Pt}$ and $\mathrm{Co} / \mathrm{Au}$ bilayers has an interfacial contribution comparable to the spin-Hall effect in the heavy-metal layer. The magnetoconductance $\Delta_{y z} g$ for the $\mathrm{Co} / \mathrm{Pt}$ bilayer is found to be of the order of a conductance quantum per interfacial atom, which exceeds the expected spin-Hall magnetoconductance by more than an order of magnitude and likely has an interfacial origin.

We thank Vivek Amin, Gerrit Bauer, Ilya Krivorotov, Farzad Mahfouzi, Branislav Nikolić, and Mark Stiles for useful discussions. This work was supported by the National Science Foundation (NSF) through Grant No. DMR1609776 (K.D.B.) and the Nebraska Materials Research Science and Engineering Center (MRSEC) Grant No. DMR1420645 (K.D.B. and A.A.K.), the US Department of Energy, Office of Science, Basic Energy Sciences Award No. DESC0014189 (A.A.K.), and EPSRC CCP9 Flagship Project No. EP/M011631/1 (M.v.S.). Calculations were performed utilizing the Holland Computing Center of the University of Nebraska, which receives support from the Nebraska Research Initiative.

\section{APPENDIX: EXPANSION OF SOT IN VECTOR SPHERICAL HARMONICS}

Any vector field $\mathbf{V}(\hat{n})$ defined on the unit sphere and tangential to it can be represented as a linear combination of orthonormal vector spherical harmonics (VSH) [52] $\mathbf{Y}_{l m}^{(\nu)}$ $(v=1,2)$,

$$
\mathbf{V}(\hat{n})=\sum_{l m v} A_{l m}^{(v)} \mathbf{Y}_{l m}^{(\nu)}(\hat{n}),
$$

where

$$
\begin{gathered}
\mathbf{Y}_{l m}^{(1)}(\hat{n})=\frac{r \nabla Y_{l m}(\hat{n})}{\sqrt{l(l+1)}}, \\
\mathbf{Y}_{l m}^{(2)}(\hat{n})=\frac{\mathbf{r} \times \nabla Y_{l m}(\hat{n})}{\sqrt{l(l+1)}} .
\end{gathered}
$$

The magnetization torque $\mathbf{T}$ induced by the applied electric field $\mathbf{E}$ is given by the torquance tensor $\hat{K}(\hat{m})$ which depends on the orientation of the magnetization $\hat{m}$,

$$
\mathbf{T}=\hat{K}(\hat{m}) \mathbf{E},
$$

and can generally be expanded in the VSH basis as

$$
\hat{K}(\hat{m})=\sum_{l m \nu} \mathbf{Y}_{l m}^{(v)}(\hat{m}) \otimes \mathbf{K}_{l m}^{(\nu)} .
$$

Here, $\mathbf{K}_{l m}^{(v)}$ are (complex) Cartesian vectors whose structure is determined by the symmetry of the system. In particular, consider the axially symmetric case ( $C_{\infty v}$ symmetry) characteristic for a polycrystalline bilayer. In this case, the torquance tensor $\hat{K}(\hat{m})$ should be invariant with respect to the rotation of the crystal around the $z$ axis. Because this rotation does not mix VSH with each other, each term in Eq. (A5) should be invariant.

If the bilayer is rotated around the $z$ axis by angle $\delta$, the VSH transforms as $\mathbf{Y}_{l m}^{(\nu)}(\theta, \phi) \rightarrow \mathbf{Y}_{l m}^{(\nu)}(\theta, \phi-\delta)=$ $e^{-i m \delta} \mathbf{Y}_{l m}^{(\nu)}(\theta, \phi)$. Therefore, $\mathbf{K}_{l m}^{(\nu)}$ must transform as $\mathbf{K}_{l m}^{(\nu)} \rightarrow$ $e^{i m \delta} \mathbf{K}_{l m}^{(\nu)}$, which is only possible for $m=0$ with $\mathbf{K}_{l 0}^{(v)} \| \hat{z}$, or for $m= \pm 1$ with $\mathbf{K}_{l, \pm 1}^{(\nu)}=K_{l, \pm 1}^{(\nu)}(\hat{x} \pm i \hat{y})$. Thus, only VSH with $m= \pm 1$ are allowed in the axially symmetric case for the torque arising in response to the in-plane electric field. We also have $K_{l,-1}^{(v)}=-K_{l, 1}^{(v) *}$, because $\hat{K}(\hat{m})$ must be real.

In this Rapid Communication we use the reference frame in which $\mathbf{E}=E \hat{x}$. Mirror reflection symmetry only allows $\operatorname{Im} \mathbf{Y}_{l 1}^{(v)}$ for odd $l$ and $\operatorname{Re} \mathbf{Y}_{l 1}^{(v)}$ for even $l$. It is then convenient to use the basis of (also orthonormal) real VSH, which are defined, similar to the real scalar spherical harmonics, as $\mathbf{Z}_{l,-1}^{(\nu)}=$ $-\sqrt{2} \operatorname{Im} \mathbf{Y}_{l 1}^{(\nu)}$ and $\mathbf{Z}_{l, 1}^{(\nu)}=-\sqrt{2} \operatorname{Re} \mathbf{Y}_{l 1}^{(\nu)}$. The torquance is then represented by the expansion

$$
\mathbf{T} / E=\sum_{l v} C_{l,(-1)^{l}}^{(v)} \mathbf{Z}_{l,(-1)^{l}}^{(v)}
$$

Apart from being orthonormal, the VSH (both complex and real) have the following useful properties for representing SOT. First, under time reversal $(\hat{m} \rightarrow-\hat{m}), \mathbf{Z}_{l m}^{(1)}$ is even for odd $l$ and odd for even $l$, while the opposite holds for $\mathbf{Z}_{l m}^{(2)}$. Further, the contribution of a given SOT term to magnetization damping is given by the curl of the effective field $\mathbf{B}=\mathbf{T} \times \hat{m}$ corresponding to that term. It follows from the definition of VSH that the effective field corresponding to the 
torque harmonic $\mathbf{Z}_{l m}^{(1)}$ has the form of $\mathbf{Z}_{l m}^{(2)}$, and vice versa. Because $\mathbf{Z}_{l m}^{(2)} \propto \hat{\mathbf{L}} Z_{l m}$, we find that the torque harmonic $\mathbf{Z}_{l m}^{(1)}$ generates damping proportional to $\hat{\mathbf{L}}^{2} Z_{l m}=l(l+1) Z_{l m}$, i.e., simply to $Z_{l m}$. On the other hand, torque harmonics $\mathbf{Z}_{l m}^{(2)}$ do not contribute to damping at all, because the corresponding effective field is a pure gradient and, therefore, has a zero curl. Conversely, the effective field corresponding to torque harmonics $\mathbf{Z}_{l m}^{(1)}$ has zero divergence. These properties make it natural to call $\mathbf{Z}_{l m}^{(1)}$ torque harmonics purely dampinglike, and $\mathbf{Z}_{l m}^{(2)}$ purely fieldlike.

The angular dependence of the current-induced magnetization damping generated by the first two dampinglike terms in the VSH expansion, $\mathbf{Z}_{1,-1}^{(1)}$ and $\mathbf{Z}_{2,1}^{(1)}$, is proportional to $m_{y}$ and $m_{x} m_{z}$, respectively $[30,44]$.
The commonly used SOT types can be represented in terms of VSH as follows:

$$
\begin{aligned}
& \text { Dampinglike: } \hat{m} \times(\hat{y} \times \hat{m})=\sqrt{8 \pi / 3} \mathbf{Z}_{1,-1}^{(1)} \\
& \text { Fieldlike: } \hat{y} \times \hat{m}=-\sqrt{8 \pi / 3} \mathbf{Z}_{1,-1}^{(2)} \\
& \text { Planar-Hall-like }[30,45]: \\
& \qquad m_{x} \hat{m} \times(\hat{z} \times \hat{m})=\sqrt{2 \pi / 3} \mathbf{Z}_{1,-1}^{(2)}+\sqrt{2 \pi / 5} \mathbf{Z}_{2,1}^{(1)} .
\end{aligned}
$$

As can be seen from Eqs. (A7)-(A9), the leading dampinglike and fieldlike SOT terms are pure VSH, while the planar-Halllike term (A9) is a linear combination of the field-like term and a damping-like VSH with $l=2$. For brevity, we retain the terms dampinglike and fieldlike for the leading terms (A7) and (A8).
[1] J. Slonczewski, J. Magn. Magn. Mater. 159, L1 (1996).

[2] L. Berger, Phys. Rev. B 54, 9353 (1996).

[3] I. M. Miron, G. Gaudin, S. Auffret, B. Rodmacq, A. Schuhl, S. Pizzini, J. Vogel, and P. Gambardella, Nat. Mater. 9, 230 (2010).

[4] L. Liu, T. Moriyama, D. C. Ralph, and R. A. Buhrman, Phys. Rev. Lett. 106, 036601 (2011).

[5] A. Manchon, J. Železný, I. M. Miron, T. Jungwirth, J. Sinova, A. Thiaville, K. Garello, and P. Gambardella, Rev. Mod. Phys. 91, 035004 (2019).

[6] H. Nakayama, M. Althammer, Y.-T. Chen, K. Uchida, Y. Kajiwara, D. Kikuchi, T. Ohtani, S. Geprägs, M. Opel, S. Takahashi, R. Gross, G. E. W. Bauer, S. T. B. Goennenwein, and E. Saitoh, Phys. Rev. Lett. 110, 206601 (2013).

[7] Y.-T. Chen, S. Takahashi, H. Nakayama, M. Althammer, S. T. B. Goennenwein, E. Saitoh, and G. E. W. Bauer, J. Phys.: Condens. Matter 28, 103004 (2016).

[8] V. P. Amin and M. D. Stiles, Phys. Rev. B 94, 104419 (2016).

[9] V. P. Amin and M. D. Stiles, Phys. Rev. B 94, 104420 (2016).

[10] K. D. Belashchenko, A. A. Kovalev, and M. van Schilfgaarde, Phys. Rev. Lett. 117, 207204 (2016).

[11] L. Zhu, D. C. Ralph, and R. A. Buhrman, Phys. Rev. Lett. 122, 077201 (2019).

[12] W. Wang, T. Wang, V. P. Amin, Y. Wang, A. Radhakrishnan, A. Davidson, S. R. Allen, T. J. Silva, H. Ohldag, D. Balzar, B. L. Zink, P. M. Haney, J. Q. Xiao, D. G. Cahill, V. O. Lorenz, and X. Fan, Nat. Nanotechnol. 14, 819 (2019).

[13] Z. Luo, Q. Zhang, Y. Xu, Y. Yang, X. Zhang, and Y. Wu, Phys. Rev. Applied 11, 064021 (2019).

[14] V. P. Amin, J. Li, M. D. Stiles, and P. M. Haney, Phys. Rev. B 99, 220405(R) (2019).

[15] A. Hönemann, C. Herschbach, D. V. Fedorov, M. Gradhand, and I. Mertig, Phys. Rev. B 99, 024420 (2019).

[16] Y.-T. Chen, S. Takahashi, H. Nakayama, M. Althammer, S. T. B. Goennenwein, E. Saitoh, and G. E. W. Bauer, Phys. Rev. B 87, 144411 (2013).

[17] J. Sinova, S. O. Valenzuela, J. Wunderlich, C. H. Back, and T. Jungwirth, Rev. Mod. Phys. 87, 1213 (2015).

[18] L. Liu, C.-F. Pai, Y. Li, H. W. Tseng, D. C. Ralph, and R. A. Buhrman, Science 336, 555 (2012).

[19] L. Liu, O. J. Lee, T. J. Gudmundsen, D. C. Ralph, and R. A. Buhrman, Phys. Rev. Lett. 109, 096602 (2012).
[20] A. G. Aronov and Y. B. Lyanda-Geller, Pis'ma Zh. Eksp. Teor. Fiz. 50, 398 (1989) [JETP Lett. 50, 431 (1989)].

[21] V. Edelstein, Solid State Commun. 73, 233 (1990).

[22] S. D. Ganichev, M. Trushin, and J. Schliemann, in Handbook of Spin Transport and Magnetism, edited by E. Y. Tsymbal and I. Žutić (CRC, Boca Raton, FL, 2011), p. 487.

[23] A. Chernyshov, M. Overby, X. Liu, J. K. Furdyna, Y. LyandaGeller, and L. P. Rokhinson, Nat. Phys. 5, 656 (2009).

[24] I. M. Miron, K. Garello, G. Gaudin, P.-J. Zermatten, M. V. Costache, S. Auffret, S. Bandiera, B. Rodmacq, A. Schuhl, and P. Gambardella, Nature (London) 476, 189 (2011).

[25] A. Manchon, H. C. Koo, J. Nitta, S. M. Frolov, and R. A. Duine, Nat. Mater. 14, 871 (2015).

[26] X. Fan, H. Celik, J. Wu, C. Ni, K.-J. Lee, V. O. Lorenz, and J. Q. Xiao, Nat. Commun. 5, 3042 (2014).

[27] D. A. Pesin and A. H. MacDonald, Phys. Rev. B 86, 014416 (2012).

[28] A. Qaiumzadeh, R. Â. A. Duine, and M. Titov, Phys. Rev. B 92 , 014402 (2015).

[29] I. A. Ado, O. A. Tretiakov, and M. Titov, Phys. Rev. B 95, 094401 (2017).

[30] K. D. Belashchenko, A. A. Kovalev, and M. van Schilfgaarde, Phys. Rev. Mater. 3, 011401 (2019).

[31] J. Kim, J. Sinha, M. Hayashi, M. Yamanouchi, S. Fukami, T. Suzuki, S. Mitani, and H. Ohno, Nat. Mater. 12, 240 (2013).

[32] R. Ramaswamy, X. Qiu, T. Dutta, S. D. Pollard, and H. Yang, Appl. Phys. Lett. 108, 202406 (2016).

[33] J. Torrejon, J. Kim, J. Sinha, S. Mitani, M. Hayashi, M. Yamanouchi, and H. Ohno, Nat. Commun. 5, 4655 (2014).

[34] X. Zhou, M. Tang, X. L. Fan, X. P. Qiu, and S. M. Zhou, Phys. Rev. B 94, 144427 (2016).

[35] L. Wang, R. J. H. Wesselink, Y. Liu, Z. Yuan, K. Xia, and P. J. Kelly, Phys. Rev. Lett. 116, 196602 (2016).

[36] F. Freimuth, S. Blügel, and Y. Mokrousov, Phys. Rev. B 90, 174423 (2014).

[37] F. Mahfouzi and N. Kioussis, Phys. Rev. B 97, 224426 (2018).

[38] S. V. Faleev, F. Léonard, D. A. Stewart, and M. van Schilfgaarde, Phys. Rev. B 71, 195422 (2005).

[39] B. K. Nikolić, K. Dolui, M. D. Petrović, P. Plecháč, T. Markussen, and K. Stokbro, in Handbook of Materials Modeling: Applications: Current and Emerging Materials, 
edited by W. Andreoni and S. Yip (Springer, Cham, 2018), pp. 1-35.

[40] I. Turek, V. Drchal, J. Kudrnovský, M. Šob, and P. Weinberger, Electronic Structure of Disordered Alloys, Surfaces and Interfaces (Kluwer, Boston, 1997).

[41] D. Pashov, S. Acharya, W. R. L. Lambrecht, J. Jackson, K. D. Belashchenko, A. Chantis, F. Jamet, and M. van Schilfgaarde, Comput. Phys. Commun. (to be published, 2019), doi: 10.1016/j.cpc.2019.107065.

[42] N. H. Long, P. Mavropoulos, D. S. G. Bauer, B. Zimmermann, Y. Mokrousov, and S. Blügel, Phys. Rev. B 94, 180406(R) (2016).

[43] G. Y. Guo, S. Murakami, T.-W. Chen, and N. Nagaosa, Phys. Rev. Lett. 100, 096401 (2008).

[44] C. Safranski, E. A. Montoya, and I. N. Krivorotov, Nat. Nanotechnol. 14, 27 (2019).

[45] J. Kim, P. Sheng, S. Takahashi, S. Mitani, and M. Hayashi, Phys. Rev. Lett. 116, 097201 (2016).
[46] M. Kawaguchi, D. Towa, Y.-C. Lau, S. Takahashi, and M. Hayashi, Appl. Phys. Lett. 112, 202405 (2018).

[47] V. L. Grigoryan, W. Guo, G. E. W. Bauer, and J. Xiao, Phys. Rev. B 90, 161412(R) (2014).

[48] S. S.-L. Zhang, G. Vignale, and S. Zhang, Phys. Rev. B 92, 024412 (2015).

[49] K.-W. Kim, K.-J. Lee, J. Sinova, H.-W. Lee, and M. D. Stiles, Phys. Rev. B 96, 104438 (2017).

[50] M. Bode, S. Heinze, A. Kubetzka, O. Pietzsch, X. Nie, G. Bihlmayer, S. Blügel, and R. Wiesendanger, Phys. Rev. Lett. 89, 237205 (2002).

[51] C. Gould, C. Rüster, T. Jungwirth, E. Girgis, G. M. Schott, R. Giraud, K. Brunner, G. Schmidt, and L. W. Molenkamp, Phys. Rev. Lett. 93, 117203 (2004).

[52] V. B. Berestetskii, E. M. Lifshitz, and L. P. Pitaevskii, Quantum Electrodynamics (Pergamon Press, Oxford, UK, 1982). 